\title{
Electromagnetic energy transfer and switching in nanoparticle chain arrays below the diffraction limit
}

\author{
Mark L. Brongersma, * John W. Hartman, and Harry A. Atwater \\ Thomas J. Watson Laboratory of Applied Physics, California Institute of Technology, Pasadena, California 91125
}

(Received 27 September 2000)

\begin{abstract}
Electromagnetic energy transfer in plasmon wires consisting of chains of closely spaced metal nanoparticles can occur below the diffraction limit by means of coupled plasmon modes. Coherent propagation with group velocities that exceed $0.1 c$ is possible in straight wires and around sharp corners (bending radius much less than wavelength of visible light). Energy transmission through chain networks is possible at high efficiencies and is a strong function of the frequency and polarization direction of the plasmon mode. Although these structures exhibit transmission losses due to heating of about $3 \mathrm{~dB} / 500 \mathrm{~nm}$, they have optical functionality that cannot be obtained in other ways at a length scale $\ll 1 \mu \mathrm{m}$.
\end{abstract}

The fundamental physical properties of nanometer-size metal particles have been intensively researched for the last hundred years. ${ }^{1,2}$ Until recently, most effort has focused on statistically large numbers of particles in disordered arrays. From this work it is well established that at the surface plasmon frequency light strongly interacts with metal particles and excites a collective electron motion, or plasmon. ${ }^{2}$ These frequencies are typically in the visible and near-infrared part of the spectrum. ${ }^{3}$ In nanometer size particles (much smaller than the wavelength of the exciting light, $\lambda$ ) plasmon excitations produce an oscillating dipole field.

Recent developments in particle synthesis and physical characterization of nanostructures have enabled the investigation of the optical properties of single nanoparticles ${ }^{4}$ and ordered arrays of closely spaced nanoparticles. In such arrays collective effects and interparticle interactions play an important role. For example, it has been demonstrated that arrays of particles under broad-beam excitation show collective behavior. ${ }^{5}$ Furthermore, numerical simulations have shown that electromagnetic (EM) energy can be transported below the diffraction limit along linear chains of closely spaced metal nanoparticles. ${ }^{6}$ This finding could have important consequences for integrated optics which faces the fundamental limitation that, for the guiding, modulation, and amplification of light, structures are needed that have dimensions comparable to the wavelength of light. ${ }^{7}$ The EM energy transport along chains of metal nanoparticles relies on the near-field electrodynamic interaction between metal particles that sets up coupled dipole or plasmon modes. ${ }^{8}$ This type of coupling is analogous to the process of resonant energy transfer, which is observed in systems that contain closely spaced optically excited atoms, molecules, or semiconductor nanocrystals. $^{9-12}$

In this paper, the dispersion relation for coupled plasmon modes is developed for a linear chain of equally spaced metal particles using an analytical model that describes the near-field EM interaction between the particles in the dipole limit. The model provides estimates for the group velocity $v_{g}$ and the attenuation coefficient $\alpha$ of the allowed coupled plasmon waves. Furthermore, the transport of EM energy through corners and tee's that consist of intersecting chains of metal nanoparticles are considered. In combination with other structures they form the basic building blocks for fully interconnected systems of complex architecture. The dependence of the behavior on the metal particle size and shape, the interparticle spacing, the polarization direction of coupled plasmon mode, and the dielectric properties of the host matrix will be discussed.

The dispersion relation for coupled plasmon modes is developed for a linear chain of equally spaced metal particles separated by a distance $d$, as is shown in the inset of Fig. 1 . Tranverse $(T)$ plasmon modes exist with dipole moments oriented perpendicular to the chain axis and longitudinal $(L)$ plasmon modes exist with dipole moments along the propagation direction, where a mode is defined by the magnitude of the induced dipole moments $p_{i, m}(t)$ in the polarization direction $i$ at all points $m$ of the chain.

The oscillating dipole electric field $\mathbf{E}_{P}$ vector consists of three terms: $\mathbf{E}_{P}=\mathbf{E}_{F}+\mathbf{E}_{M}+\mathbf{E}_{R}$, where $\mathbf{E}_{F}$ is proportional to

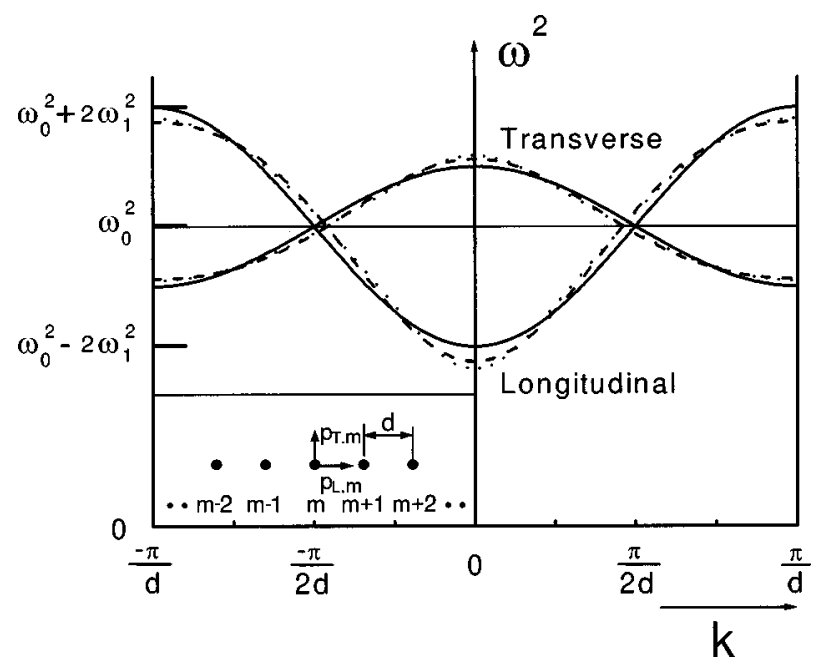

FIG. 1. Dispersion relation for plasmon modes in a linear chain of metal nanoparticles, depicted in the inset, including the twofold degenerate branch of the transverse modes and the longitudinal mode. The calculation is performed for nearest-neighbor interactions only (solid curve), next-nearest-neighbor interactions (dashed curve), and fifth-nearest-neighbor interactions (dotted curve). 
$R^{-3}, \mathbf{E}_{M}$ to $R^{-2}$, and $E_{R}$ to $R^{-1}$, where $R$ is the distance from the dipole. ${ }^{12}$ The term $E_{R}$ describes the radiation field and dominates when $R \gg \lambda$. The Förster field $\mathbf{E}_{F}$ is dominant in the quasistatic limit, $R \ll \lambda$. For this reason, the description of the interaction between two closely spaced dipoles $(d$ $\ll \lambda$ ) only involves the Förster field. An oscillating dipole $p_{i, m}$ at a point $m$ in the chain thus produces an electric field $E_{i, m}$, at the neighbor locations $m-1$ and $m+1$, that is given by

$$
E_{i, m}(t)=\frac{\gamma_{i} p_{i, m}(t)}{4 \pi \varepsilon_{0} n^{2} d^{3}}
$$

in which $\gamma_{i}$ is a polarization-dependent constant for which $\gamma_{T}=1$ and $\gamma_{L}=-2, \varepsilon_{0}$ is the free space permittivity, and $n$ is the refractive index of the host material. ${ }^{12}$ Because of the $1 / d^{3}$ dependence of $E_{i, m}$ nearest-neighbor interactions are dominant. We quantify the effects of the next-nearestneighbor and more distant neighbor dipole interactions, but for the moment assume only nearest-neighbor interactions. For a chain of Hertzian dipoles, ${ }^{13}$ where $p_{i, m}=q x_{i, m}$, for a charge $q$, and $x_{i, m}$ its distance from equilibrium in the $i$ direction at point $m$ in the chain, the equation of motion is

$\ddot{p}_{i, m}=-\omega_{0}^{2} p_{i, m}-\Gamma_{I} \dot{p}_{i, m}+\frac{\Gamma_{R}}{\omega_{0}^{2}} \dddot{p}_{i, m}-\gamma_{i} \omega_{1}^{2}\left(p_{i, m-1}+p_{i, m+1}\right)$.

The first term describes dipole eigenmotion at angular resonance frequency $\omega_{0}$ and the second term and third term model the damping of plasmon waves along the chain. The constant $\Gamma_{l}$ is the electronic relaxation frequency due to interactions with phonons, electrons, lattice defects, and impurities and $\Gamma_{R}$ is the relaxation frequency due to radiation into the far field. ${ }^{14}$ The fourth term incorporates the electrodynamic interaction with the nearest-neighbor dipoles at $m$ -1 and $m+1$. This term is responsible for the existence of propagation wave solutions. The coupling strength is determined by the value of $\omega_{1}^{2}$, which is given by $\omega_{1}^{2}$ $=q e / 4 \pi m^{*} \varepsilon_{0} n^{2} d^{3}$, where $q$ is the magnitude of the oscillating charge, $m^{*}$ is the optical effective electron mass, and $e$ is the electron charge. For an array of $25 \mathrm{~nm}$ radius $\mathrm{Ag}$ particles spaced by $75 \mathrm{~nm}$ in vacuum $(n=1)$ the value of $\omega_{1}=1.4 \times 10^{15} \mathrm{rad} / \mathrm{s}^{-1}$. For this calculation we have used a charge $q=6.1 \times 10^{-13} \mathrm{C}\left[\rho_{\mathrm{el}}=5.85 \times 10^{22} / \mathrm{cm}^{3}\right.$ (Ref. 15) $]$ and the $m^{*}=8.7 \times 10^{-31} \mathrm{~kg}$ for $\mathrm{Ag} .{ }^{16}$ The value of $\omega_{1}$ is of the same magnitude as $\omega_{0} \approx 5 \times 10^{15} \mathrm{rad} / \mathrm{s}$ in vacuum, indicative of a strong interparticle coupling.

Propagating wave solutions to Eq. (2) are of the form:

$$
p_{i, m}=P_{i, 0} \exp [-\alpha m d+i(\omega t \pm k m d)],
$$

where $(\omega t-k m d)$ is appropriate when the phase and group velocity are parallel and $(\omega+k m d)$ is appropriate when the phase and group velocity are antiparallel. The value of $P_{i, 0}$ determines the dipole moment at $m=0$ in the direction $i$. The damping of the plasmon wave per unit length is given by the attenuation coefficient $\alpha$. The angular frequency and wave vector of the plasmon wave are given by $\omega$ and $k$, respectively. Substitution of Eq. (3) into Eq. (2) and equating real and imaginary parts yields

$$
\begin{gathered}
\omega^{2}=\omega_{0}^{2}+2 \gamma_{i} \omega_{1}^{2} \cos (k d) \cosh (\alpha d), \\
0=\omega \Gamma_{I}+\frac{\omega^{3} \Gamma_{R}}{\omega_{0}^{2}}+2 \gamma_{i} \omega_{1}^{2} \sin (k d) \sinh (\alpha d) .
\end{gathered}
$$

Equation (4) is the dispersion relation for the plasmonpolariton waves. For small damping, $\alpha d \ll 1$, Eq. (4) reduces to

$$
\omega^{2}=\omega_{0}^{2}+2 \gamma_{i} \omega_{1}^{2} \cos (k d) .
$$

The solid lines in Fig. 1 show the dispersion relations in Eq. (6) for $T$ and $L$ modes. Also plotted are dispersion curves calculated including next-nearest-neighbor interactions (dashed curves), and up to fifth-nearest-neighbor interactions (dotted curves). It is clear that nearest-neighbor interactions are most significant for the transport properties of the nanoparticle chains. The bandwidth of the $L$ branch $\Delta \omega_{L}$, is larger than that of the twofold degenerate $T$ branch, $\Delta \omega_{T}$. The group velocity of these waves is given by $v_{g, i}$ $=d \omega / d k=\left(d \omega^{2} / d k\right) /\left(d \omega^{2} / d \omega\right)=\gamma_{i} \omega_{1}^{2} d \sin (k d) / \omega$. Since the $d \omega^{2} / d k$ is the slope of the dispersion curves in Fig. 1, it can easily be seen that at a given $\omega$ the $L$ waves propagate faster than the $T$ waves. At resonance the group velocities for both the $L$ wave and the $T$ wave are maximum and $v_{g, L}$ $=2 v_{g, T}$. This results from the stronger EM coupling for $L$ waves than for $T$ waves.

For an array of $25 \mathrm{~nm}$ radius Ag particles spaced by 75 $\mathrm{nm}$ in vacuum $(n=1)$, we find that the group velocities at resonance are $v_{g, T}=2.9 \times 10^{7} \mathrm{~m} / \mathrm{s}$ and $v_{g, L}=5.8 \times 10^{7} \mathrm{~m} / \mathrm{s}$. These values are about two orders of magnitude higher than the saturation velocities of electrons in semiconductors. ${ }^{17}$ For these parameters, the corresponding bandwidths are $\Delta \omega_{T}=7.9 \times 10^{14} \mathrm{~s}^{-1}(520 \mathrm{meV})$ and $\Delta \omega_{L}=1.6 \times 10^{15} \mathrm{~s}^{-1}$ $(1.05 \mathrm{eV})$. Because of the large bandwidths, the plasmon wave group velocity is a slowly varying function of $\omega$ near $\omega_{0}$, i.e., the dispersion is minimal.

In the regime of small damping, Eq. (5) can be rewritten as

$$
\alpha=\frac{\Gamma_{I}+\left(\omega^{2} / \omega_{0}^{2}\right) \Gamma_{R}}{2 v_{g}},
$$

showing that the attenuation of the plasmon-polariton wave is given by the ratio of the total decay rate divided by two times the group velocity of the wave. The attenuation of the signal intensity is given by $2 \alpha$. The magnitude of $\Gamma_{I}$ is determined by the mean free path of the electrons in the metal nanoparticles. The mean free path of an electron in a nanoparticle is reduced compared to its bulk value $\lambda_{B}$ due to inelastic collisions with the particle surface. Under the assumption that the collision processes are independent, the relaxation rates can be added according to Matthiessen's rule $^{18}$ and $\Gamma_{I}=v_{F} / \lambda_{B}+C v_{F} / R$, in which $C$ is a constant of order 1. ${ }^{19}$ For $\operatorname{Ag~} v_{F}=1.38 \times 10^{6} \mathrm{~m} / \mathrm{s}$ and $\lambda_{B}=57 \mathrm{~nm} .{ }^{20}$ When we assume $C$ to be 1 , the value of $\Gamma_{I}$ for a $25 \mathrm{~nm}$ radius $\mathrm{Ag}$ particle is $\Gamma_{I}=7.9 \times 10^{13} \mathrm{~s}^{-1}$, corresponding to a 13 fs decay time. Such a decay time accords with femtosecond decay-time measurements of $10 \mathrm{fs}$ on $\mathrm{Ag}$ nanoparticles. $^{21}$ The value of $\Gamma_{R}$ is given by $\Gamma_{R}=\omega_{0}^{2} \tau$, where $\tau$ is a constant given by $\tau=2 e^{2} / 3 m^{*} c^{3}$ $=6.26 \times 10^{-24} \mathrm{~s}^{-1} \cdot{ }^{14}$ For an $\omega_{0} \approx 5 \times 10^{15} \mathrm{rad} / \mathrm{s}$ the value of 
$\Gamma_{R}=1.6 \times 10^{8} \mathrm{~s}^{-1}$, corresponding to a $6.4 \mathrm{~ns}$ decay time. Since $\Gamma_{I}$ is about 5 orders of magnitude higher than $\Gamma_{R}$, radiation losses can be neglected. On the other hand, transmission losses and heating of these wires due to inelastic collisions is a serious consideration. The value of $\alpha$ at resonance for our standard example of a longitudinal wave propagating along a chain of $25 \mathrm{~nm} \mathrm{Ag}$ particles spaced by $75 \mathrm{~nm}$ in vacuum is $\alpha=6.8 \times 10^{5} \mathrm{~m}^{-1}$, corresponding to a decay length of $1.5 \mu \mathrm{m}$ or 20 interparticle spacings. Based on this value of $\alpha$, the attenuation coefficient for the signal intensity of a longitudinal wave along this wire is $2 \alpha=1.4$ $\times 10^{6} \mathrm{~m}^{-1}$, corresponding to $3 \mathrm{~dB} / 500 \mathrm{~nm}$. This value is in close agreement with the numerical calculation from Quinten et al. that predicted a signal attenuation coefficient of $2 \alpha$ $=1.1 \times 10^{6} \mathrm{~m}^{-1},{ }^{6}$ corresponding to $2.4 \mathrm{~dB} / 500 \mathrm{~nm}$. The negative effect of a quite severe attenuation is partly counteracted by the resonant enhancement of the EM field near the first particle in the chain. ${ }^{6}$ Furthermore, it should be realized that the nanoscale dimensions of plasmon wires can have electromagnetic functionality that cannot be obtained in other ways at a length scale $\ll 1 \mu \mathrm{m}$.

The EM energy transport through corners and tee junctions can be calculated by requiring continuity of the plasmon waves and conservation of energy flux at the intersection of the chains. In general, part of the incident wave flows through the structure and part is reflected. The efficiency of the power transmission depends on the geometry of the structure, the frequency, and polarization directions of the plasmon waves that enter and exit a structure. In this paper only structures that consist of chains and $90^{\circ}$ corners are considered. This greatly simplifies the modeling since in that case only three situations need to be distinguished: (1) in a turn an $L$ wave transforms into a $T$ wave, (2) in a turn a $T$ wave transforms into an $L$ wave, and (3) in a turn a $T$ wave remains a $T$ wave. At certain values of $\omega$ the importance of the frequency and polarization direction is manifested in the dispersion relation showing only $L$ modes can exist and $T$ modes are forbidden. This implies that a $90^{\circ}$ corner in which $L$ waves are transformed into $T$ waves acts as a frequency or polarization filter. It can also be shown that the transmission efficiency through any structure is maximum at $\omega=\omega_{0}$ (Ref. 22 ) and in the work here the value of $\omega_{0}$ is assumed. Figure 2 shows calculated power transmission coefficients, $\eta$, in the nearest-neighbor approximation for all realizable $90^{\circ}$ corner and tee structures. An $\eta$ value of 1 corresponds to $100 \%$ transmission. Notice that all structures have $\eta$ values exceeding 0.64 , showing that power flow around $90^{\circ}$ corners with dimensions much smaller than the wavelength of light is possible at high efficiencies. This is impossible in conventional dielectric waveguide technology, ${ }^{7}$ and is more akin to certain photonic crystal structures. ${ }^{23}$ Beyond the nearestneighbor dipole-dipole interaction approximation more detailed analysis of plasmon wire structures can be made using full field EM simulations using, e.g., finite difference time domain calculations, which will be reported. ${ }^{22}$

A plasmon switch or inverter consisting of a tee structure is pictorially shown in the inset of Fig. 3. It shows the incident signal $\mathrm{S}$ and modulation $\mathrm{M}$ waves and an outgoing transmitted $\operatorname{Tr}$ wave. When the $\mathrm{S}$ and $\mathrm{M}$ waves have the same polarization and possess opposite phases they destructively interfere. For a specific ratio of the power in the $\mathrm{S}$

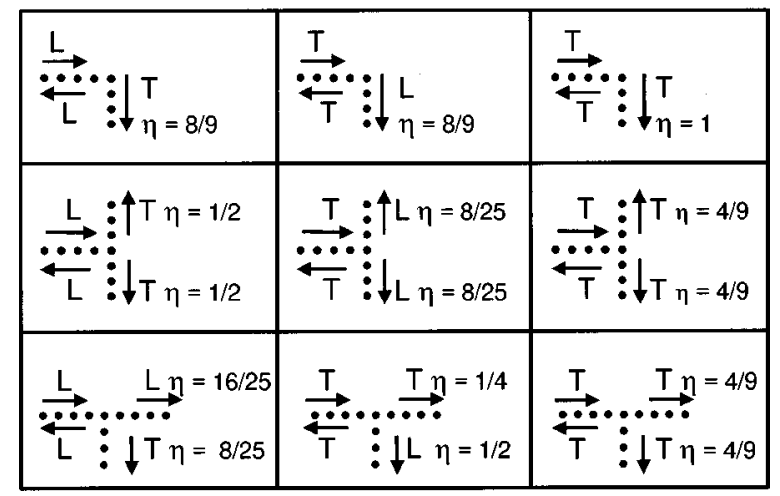

FIG. 2. Calculated power transmission coefficient $\eta$ for nanoparticle chain-array corner and tee structures with $90^{\circ}$ corners. The arrows indicate the direction of the power flow of longitudinal waves $(L)$ and transverse waves $(T)$. An $\eta$ value of 1 corresponds to $100 \%$ transmission.

wave, $P_{S}$, to the power in the $\mathrm{M}$ wave, $P_{M}$, the power in the Tr wave, $P_{\mathrm{Tr}}$, can be made zero, in other words the switch is in the off state. Figure 3 shows the dependence of the normalized transmitted power $P_{T r} / P_{S}$ as a function of the normalized modulation power $P_{M} / P_{S}$ for the case of a longitudinal $\mathrm{S}$ wave and a transverse $\mathrm{M}$ wave. At zero $P_{M}$, the value of $P_{T r} / P_{S}$ is determined by the transmission efficiency of the structure for longitudinal $S$ waves. When $P_{M} / P_{S}$ is 2 the switch is in the off-state and for higher $P_{M} / P_{S}$ the transmitted power is dominated more and more by the power in the $M$ wave and the dependence of $P_{T r} / P_{S}$ on $P_{M} / P_{S}$ becomes linear. The differential power gain, $d P_{\mathrm{Tr}} / d P_{M}$, as a function of $P_{M} / P_{S}$ is obtained by differentiation of the curve in Fig. 3. It is negative below $P_{M} / P_{S}=2$ and positive for larger values.

The transport properties of plasmon wire and network structures are dependent on the physical properties of the metal particles and the host matrix. The noble metals $\mathrm{Ag}, \mathrm{Cu}$, and $\mathrm{Au}$ have a long mean free path of electrons, which minimizes the attenuation of plasmon waves due to resistive heat-

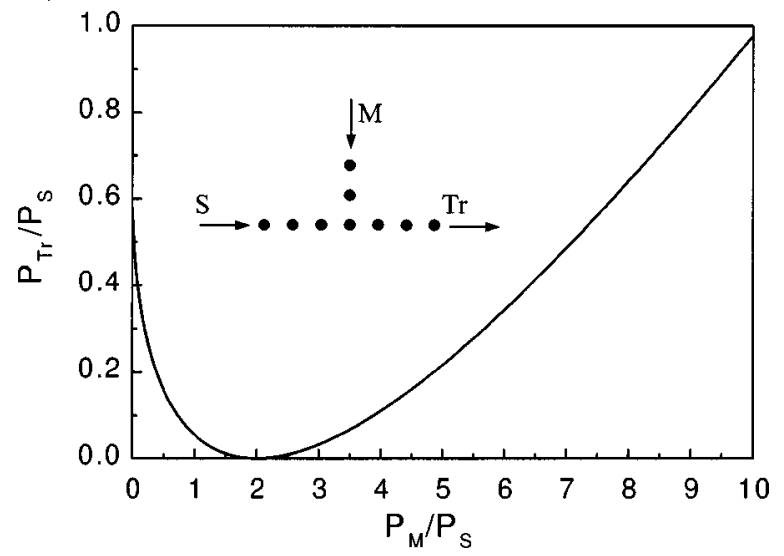

FIG. 3. Normalized transmitted power, $P_{T r} / P_{S}$, as a function of the normalized modulation power, $P_{M} / P_{s}$ of the switch depicted in the inset. The switch consists of two orthogonal chains of metal nanoparticles. The specific functional dependence is valid for a longitudinal signal plasmon wave $S$, a longitudinal transmitted plasmon wave $T_{r}$, and a transverse the modulation plasmon wave that is out of phase with $S$. 
ing. Particle sizes larger than the mean free path show a reduced effect of inelastic electron scattering from the metal particle/host matrix compared to smaller ones, but larger interparticle spacing reduces the confinement of the EM energy to the chain and reduces $v_{g}$. Particle shape also plays an important role. For example, since ellipsoids can be spaced closer together than spheres of the same volume the group velocity along a chain of ellipsoids can be made higher than for a chain of spheres of equivalent volume. The choice of host matrix can matter in three ways. First, a poor choice of host can result in significant damping of the plasmon wave because the plasmons can couple to various degrees of freedom in the host. ${ }^{24}$ Second, by varying the refractive index $n$ of the matrix, the resonance wavelength can be shifted over several hundred $\mathrm{nm}$ from the visible to the near

*Email address: mbrong@caltech.edu

${ }^{1}$ G. Mie, Ann. Phys. (Leipzig) 25, 377 (1908).

${ }^{2}$ U. Kreibig and M. Vollmer, Optical Properties of Metal Clusters (Springer-Verlag, Berlin, 1994), p. 23.

${ }^{3}$ D. D. Nolte, J. Appl. Phys. 76, 3740 (1994).

${ }^{4}$ T. Klar et al., Phys. Rev. Lett. 80, 4249 (1998).

${ }^{5}$ J. R. Krenn et al., Phys. Rev. Lett. 82, 2590 (1999).

${ }^{6}$ M. Quintin, A. Leitner, J. R. Krenn, and F. R. Aussenegg, Opt. Lett. 23, 1331 (1998).

${ }^{7}$ B. E. A. Saleh and M. C. Teich, The Fundamentals of Photonics (Wiley, New York, 1991), p. 238.

${ }^{8}$ J. R. Krenn et al., Phys. Rev. Lett. 82, 2590 (1999).

${ }^{9}$ Th. Förster, Ann. Phys. (Leipzig) 2, 55 (1948).

${ }^{10}$ D. S. Citrin, Opt. Lett. 20, 901 (1995).

${ }^{11}$ D. L. Dexter, J. Chem. Phys. 21, 836 (1953).

${ }^{12}$ B. W. van der Meer, G. Coker III, and S.-Y. S. Chen, Resonance Energy Transfer (Wiley, New York, 1994), p. 35.

${ }^{13}$ Walter Greiner, Classical Electrodynamics (Springer-Verlag, infrared. ${ }^{3}$ Third, an increase in $n$ of the matrix decreases the coupling strength between the particles and thus reduces $v_{g}$.

To date, we have performed experiments using nanophotonic analog structures that operate in the microwave frequency regime $(8 \mathrm{GHz}){ }^{25}$ For example, for structures with an interparticle spacing of $0.05 \lambda$, the transmitted intensities around both sharp corners is about $4 \mathrm{~dB}$, in close agreement with the results of microwave device simulations.

In conclusion, we have developed a general framework for analysis of plasmon wire structures with characteristic dimensions $\leqslant 0.1 \lambda$ in which EM energy can be transported below the diffraction limit, with high efficiencies, and at group velocities $>0.1 \mathrm{c}$.

We would like to acknowledge S. A. Maier and K. J. Vahala for useful discussions. This work was supported by the NSF.

New York, 1996), p. 429.

${ }^{14}$ J. D. Jackson, Classical Electrodynamics, 2nd ed. (Wiley, New York, 1962), p. 782.

${ }^{15}$ C. Kittel, Introduction to Solid State Physics (Wiley, New York, 1986), p. 134.

${ }^{16}$ P. B. Johnson and R. W. Christy, Phys. Rev. B 12, 4370 (1972).

${ }^{17}$ S. M. Sze, Physics of Semiconductor Devices (Wiley, New York, 1981), p. 44.

${ }^{18}$ N. W. Ashcroft and N. D. Mermin, Solid State Physics (Saunders College, Philadelphia, 1976), p. 323.

${ }^{19}$ U. Kreibig and L. Geinzel, Surf. Sci. 156, 678 (1985).

${ }^{20}$ K. Uchida et al., J. Opt. Soc. Am. B 11, 1236 (1994).

${ }^{21}$ B. Lamprecht, A. Leitner, and F. R. Aussenegg, Appl. Phys. B: Lasers Opt. 64, 269 (1997).

${ }^{22}$ M. L. Brongersma et al. (unpublished).

${ }^{23}$ Attila Mekis et al., Phys. Rev. Lett. 77, 3787 (1996).

${ }^{24}$ B. N. J. Persson, Surf. Sci. 281, 153 (1993).

${ }^{25}$ S. A. Maier, M. L. Brongersma, and H. A. Atwater (unpublished). 